deutlich, dass über ein Viertel der Vorlagen (sechs von 22) ohne Aussprache angenommen wurde $^{13}$, während es lediglich bei einem dieser Projekte, nämlich beim Vertragsarztrechtsänderungsgesetz, zu einer namentlichen Abstimmung kam, die als Indiz für eine überdurchschnittlich hohe Konfliktintensität interpretiert werden kann.

Daraus folgt, dass die einfache Zählung der nicht mehr zustimmungsbedürftigen Gesetze die Vergrößerung des Handlungsspielraums der Bundesregierung mit hoher Wahrscheinlichkeit überschätzt. Insofern wird die Wissenschaft noch etwas Geduld benötigen, bis sich feststellen lässt, ob die Föderalismusreform I auch bei kontroversen Gesetzen (und unter den Bedingungen abweichender parteipolitischer Mehrheiten in Bundestag und Bundesrat) zu einer Reduzierung der Vetomacht des Bundesrates beigetragen hat.

13 „Bei den ohne Debatte beschlossenen Gesetzen handelt es sich meist um spezielle Anpassungsgesetze von marginaler Bedeutung und um Vertragsgesetze“, Wolfgang Ismayr, Gesetzgebung im politischen System Deutschlands, in: ders. (Hrsg.), Gesetzgebung in Westeuropa. EU-Staaten und Europäische Union, Wiesbaden 2008, S. 403.

\title{
Die (gemäßigte) Mehrheitswahl im Streit der Wissenschaft. Eine Antwort auf Frank Decker, Harald Schoen und Stefan Köppl
}

\author{
Gerd Strohmeier
}

Der mit meinem Beitrag in Heft 3/2007 der ZParl unternommene Versuch, die „eingeschlafene“ Wahlrechtsdebatte „aufzurütteln“ (S. 579), ist gelungen. Dies zeigen neben Reaktionen von Politikern und Journalisten auch die Repliken von Frank Decker ${ }^{1}$ und Harald $S_{\text {shoen }}^{2}$ in Heft 4/2007 und von Stefan Köpp ${ }^{3}$ in Heft 1/2008 der ZParl. Die Kritik an meinem Beitrag überraschte keineswegs, ihr Gegenstand allerdings sehr: höchst unterschiedliche Positionen, die sich zum Teil widersprechen, aber nicht oder nur am Rande auf die zentralen Argumente für die („gemäßigte“) Mehrheitswahl eingehen - und diese offensichtlich auch nicht (in größerem Umfang) in Frage stellen.

Mein Beitrag enthält in der Tat „eine klare normative Präferenz [...] für die Mehrheitswahl“ (Decker, S. 857). Diese wurde unter anderem damit begründet, dass die Mehrheitswahl Situationen der Unregierbarkeit sowie dem Zwang zur Bildung einer Großen Koalition entgegenwirkt. Dabei wird die Fähigkeit zur Regierungsbildung keineswegs - wie

1 Frank Decker, Konsens- oder mehrheitsdemokratischer Wandel des Parlamentarismus? Eine Replik auf Gerd Strohmeier in Heft 3/2007 der ZParl, in: ZParl, 38. Jg. (2007), H. 4, S. 857 - 861.

2 Harald Schoen, Eine optimale Lösung? Eine Replik auf Gerd Strohmeier in Heft 3/2007 der ZParl, in: ZParl, 38. Jg. (2007), H. 4, S. $862-865$.

3 Stefan Köppl, Ein Blick über die Grenzen hilft manchmal weiter. Eine Replik auf Gerd Strohmeier in Heft 3/2007 der ZParl, in: ZParl, 39. Jg. (2008), H. 1, S. 163 - 168. 
Decker (S. 858) behauptet - mit Regierungsfähigkeit gleichgesetzt. Zwar hängt diese oder - besser - die Reform- und Steuerungsfähigkeit vom Regierungstyp beziehungsweise der Anzahl der Regierungsparteien $\mathrm{ab}^{4}$, doch habe ich diesen Zusammenhang in meinem Beitrag kaum diskutiert (obwohl er ein zusätzliches Argument für die Mehrheitswahl gewesen wäre). Die Reformfähigkeit wird nur bei der Darstellung verschiedener (möglicher) Nachteile Großer Koalitionen thematisiert. Dabei wird deutlich gemacht, dass Große Koalitionen unter Umständen „- bei schlechter interner Kooperation - aufgrund ,regierungsinterner' Reformblockaden nur durch Minimalkompromisse regieren“" (S. 582), jedoch nicht „die seit 2005 amtierende Große Koalition apodiktisch als Ursache von Politikblockaden und Reformstillstand gewertet" (Decker, S. 858). Da Decker die Wahlrechtsdiskussion auf eine Reformstaudiskussion reduziert, verwundert es nicht, dass er ausführlich die Rolle des Bundesrats thematisiert. Die „Existenz des Vetospielers Bundesrat“ (Decker, S. 858) oder - besser - die Existenz des Bundesrats als Vetospieler (dies ist er nämlich nur bei zustimmungsbedürftigen Gesetzen) ${ }^{5}$ wurde von mir - wie Decker (S. 858) richtig feststellt bewusst ausgeblendet; allerdings nur aus einem Grund: um nicht zu sehr von der eigentlichen Argumentationslinie abzuschweifen.

Nach Decker ist das Problem der „gegenläufigen Mehrheitsverhältnisse zwischen Bundestag und Bundesrat“ als die „Ursache für Politikblockaden“ „durch das Zusammengehen der beiden großen Parteien entschärft worden“ (S. 858). Diese Argumentation ist aus mindestens drei Gründen problematisch:

Erstens müssen sich die großen Parteien - wie von mir (S. 582) erläutert - gegeneinander profilieren. Dies erfolgt bei Großen Koalitionen im Kern nur an einem anderen Ort und in einer anderen Art als bei kleinen Koalitionen mit unterschiedlichen Mehrheiten in Bundestag und Bundesrat. Mit der Bildung der zweiten Großen Koalition hat sich weniger der Wettbewerb zwischen den großen Parteien als vielmehr die Arena und die Austragung dieses Wettbewerbs verändert: Die wesentliche Konfliktlinie verläuft nicht mehr zwischen Bundestag und Bundesrat, sondern zwischen den Regierungsparteien, und der wesentliche Verhandlungsort ist nicht mehr der Vermittlungs-, sondern der Koalitionsausschuss. Dass dies ein erfolgreicher(er) Verhandlungsort für die großen Parteien sein kann, habe ich nicht bestritten. Dass dies nicht so sein muss, zeigen einige Beispiele. Die Kooperation in einer Großen Koalition unterscheidet sich aufgrund des spezifischen Wettbewerbsverhältnisses zwischen den großen Parteien jedenfalls deutlich von jener in einer kleinen und hängt zudem auch äußerst stark von situativen Ereignissen, Meinungsumfragen, anstehenden Landtagswahlen und der nächsten Bundestagswahl ab.

Zweitens darf nicht davon ausgegangen werden, dass eine Große Koalition stets eine (stabile sowie breite) Mehrheit im Bundesrat hat. Berücksichtigt man bei der Analyse der Verabschiedung zustimmungsbedürftiger Gesetze die spezifischen Abstimmungsmodalitä-

4 Gerd Strohmeier, Vetospieler - Garanten des Gemeinwohls und Ursachen des Reformstaus, Baden-Baden 2005, S. 70 ff. Eine Einparteiregierung ist grundsätzlich handlungsfähiger als eine Koalitionsregierung, auch wenn die Regierungspartei „mit Zerreißproben zwischen verschiedenen Flügeln zu kämpfen [hat]“ (Köppl, S. 167) - die es in Parteien übrigens auch bei einer Koalitionsregierung gibt.

5 George Tsebelis, Decision Making in Political Systems: Veto Players in Presidentialism, Parliamentarism, Multicameralism and Multipartyism, in: British Journal of Political Science, 25. Jg. (1995), S. 289 - 325, S. 305. 
ten im Bundesrat ${ }^{6}$ und legt man bei der Erfassung der Mehrheitsverhältnisse im Bundesrat das ROM-Modell ${ }^{7}$ zugrunde, hatte die zweite Große Koalition unmittelbar nach dem Regierungswechsel von 2005 nur eine äußerst knappe Mehrheit im Bundesrat: zwei Stimmen beziehungsweise ein Land. ${ }^{8}$ Ferner ist anzumerken, dass der Gegenpol „Bund und Länder“ bei unterschiedlichen Mehrheiten in Bundestag und Bundesrat nicht einfach - wie Decker es tut (S. 859) - mit dem Gegenpol „Regierung und Opposition“ gleichgesetzt werden kann und der Bundesrat nicht vollends auf einen Spielball parteipolitischer Interessen reduziert werden darf.

Drittens ist das „Blockadeproblem“ zwischen Bundestag und Bundesrat langfristig beziehungsweise strukturell durch die am 1. September 2006 in Kraft getretene Föderalismusreform „entschärft“ worden. Ob der Erfolg dieser Reform groß, mittelmäßig oder nur gering war, hängt letztlich von der Perspektive des Betrachters ab. Es ist jedoch völlig überzogen, wenn Decker (S. 860) behauptet, dass ihr „kein Erfolg beschieden war“. Sie hat jedenfalls bewirkt, dass nun (unabhängig vom Regierungstyp) grundsätzlich weniger Gesetze der Zustimmung des Bundesrats bedürfen. ${ }^{9}$ Dass bei der Einführung der Mehrheitswahl auf Bundesebene in den Ländern der Druck entstünde, „die eigenen Wahlrechtsregelungen jenen des Bundes anzupassen“, dies „mit ziemlicher Sicherheit abweichende Mehrheitsverhältnisse zur Folge [hätte] “ und bei „einfarbigen Mehrheiten [...] die Kompromissbildung [...] noch schwieriger [wäre] “ (Decker, S. 858), ist eine Kette von Spekulationen, die keinesfalls eintreten müsste und in dieser Form wohl auch nicht eintreten würde. Warum sollte auch zum Beispiel bei einfarbigen Mehrheiten die Kompromissbildung zwischen Bundestag und Bundesrat schwieriger sein? Wieso sollten Konflikte zwischen Bundestag und Bundesrat durch eine kleine Regierungspartei „abgemildert“ (Decker, S. 859) - und nicht verstärkt - werden?

In meinem Beitrag wird die gemäßigte Mehrheitswahl als „optimale Lösung“ bezeichnet, da sie „den Vorteil der Mehrheitswahl [...] impliziert und den Nachteil der Mehrheitswahl [...] reduziert"10 (S. 586). Dass das vorgeschlagene Wahlsystem trotz der „proportionalen Komponente“ „kleine Parteien im Bundestag empfindlich zugunsten großer Parteien schwächt“ (Schoen, S. 865), ist der „Preis für den mehrheitsbildenden Effekt" (Strohmeier, S. 589). Es ist eben völlig unmöglich, ein Wahlsystem zu schaffen, das nur Vor- und keine Nachteile hat. Seriöse und sachkundige Vertreter der „Mehrheitswahl mit proportionaler Zusatzliste“ würden nie behaupten, damit das „Beste aus zwei Welten“ (Köppl, S. 166) erreichen zu können. Die grundsätzlich existierende Diskrepanz zwischen dem Repräsentationsziel der Mehrheitswahl und dem der Verhältniswahl verlangt nach einer norma-

6 Beschlüsse mit absoluter Mehrheit, einheitliche Stimmabgabe der Länder, negative Wirkung von Enthaltungen.

7 Gerd Strohmeier, Der Bundesrat: Vertretung der Länder oder Instrument der Parteien?, in: ZParl, 35. Jg. (2004), H. 4, S. $717-731$.

8 Wäre nach der Bürgerschaftswahl 2003 in Bremen keine Große Koalition, sondern eine - rechnerisch mögliche - rot-grüne Koalition gebildet worden, hätte die Große Koalition zunächst keine Mehrheit im Bundesrat gehabt.

9 Vgl. die Angaben des Bundesministeriums des Innern (http://www.bmi.bund.de, 23. Oktober 2007) sowie den Beitrag von Reimut Zohlnhöfer in diesem Heft der ZParl.

10 Vor diesem Hintergrund ist es völlig unverständlich, wie Köppl (S. 166) in dem vorgeschlagenen Wahlsystem das Ziel einer „Verschmelzung von Union und FDP beziehungsweise SPD und Grünen“ erkennen kann. 
tiv begründeten Entscheidung - die in meinem Beitrag zugunsten der Mehrheitswahl ausfällt.

Durch die dort vorgeschlagene Reform würden kleine Parteien nicht gemäß ihres Stimmenanteils im Bundestag repräsentiert sein und „nicht den Fraktionsstatus erreichen“ (Schoen, S. 862). „Selbst wenn die Gruppen den Fraktionen rechtlich gleichgestellt würden“ (ebenda, S. 863), wäre es diesen wohl nicht möglich, „in allen Gremien des Bundestags wettbewerbsfähig mitzuwirken und den Fraktionen Paroli zu bieten“ (ebenda, S. 863). Allerdings wäre es der Opposition - im Gegensatz zur Opposition bei einer Großen Koalition - möglich, der Regierung Paroli zu bieten. So könnte die große Oppositionspartei „eine Sondersitzung des Bundestags einberufen oder eine abstrakte Normenkontrolle beantragen“" (Strohmeier, S. 582). Sicherlich könnte die Einführung der Mehrheitswahl, wie von Schoen (S. 863) behauptet, von der Öffentlichkeit oder - genauer - von Teilen der Öffentlichkeit dennoch als „Machtmissbrauch“ oder als Widerspruch zu tradierten „Repräsentations- und Gerechtigkeitsvorstellungen“ verstanden werden. Wie aber wird eine schwarzrote Koalition von den Wählern verstanden, die bei der Bundestagswahl 2005 entweder für eine schwarz-gelbe oder eine rot-grüne Koalition votierten? Dabei ist zu bedenken, dass das Hauptaugenmerk bei Wahlen auf die Bestellung der Regierung und damit die Repräsentation der Parteien auf der Regierungsebene gerichtet ist. Auf dieser Ebene würden die kleinen Parteien durch die vorgeschlagene Wahlrechtsreform ihre Rolle als Mehrheitsbeschaffer oder -verhinderer und „übermächtiger Mitregent“ verlieren. Damit wären sie jedoch nicht „zur Bedeutungslosigkeit verurteilt“ (Decker, S. 859). Schließlich hat die Opposition eine „wichtige Rolle bei der Thematisierung und Entwicklung von Lösungen“ (Köppl, S. 167). Allerdings darf diese Rolle nicht, wie $K o ̈ p p l(S .166$ f.) es tut, überschätzt beziehungsweise annähernd mit der einer Regierungsfraktion gleichgesetzt werden. Eine kleine Regierungsfraktion hat deutlich mehr Einfluss als eine kleine Oppositionsfraktion. Dieser beruht „im Wesentlichen auf ihrer Vetoposition" (Köppl, S. 167). Dabei handelt es sich jedoch nicht um eine - wie Köppl (S. 167) offensichtlich denkt - x-beliebige, sondern um eine ganz entscheidende Position. Natürlich übt auch eine große Regierungspartei einen überproportional großen Einfluss aus (Köppl, S. 167). In einer Konkurrenzdemokratie, in der grundsätzlich nicht alle (wesentlichen) im Parlament repräsentierten Parteien in der Regierung vertreten sind, ist dies bei Regierungsparteien immer der Fall. Fraglich ist jedoch, in welchen Relationen sich dieser Einfluss ergibt und wie er sich argumentativ rechtfertigen lässt. Am besten ist dies bei einer Einparteiregierung möglich (Strohmeier, S. 583 ff.) ${ }^{11}$, auch wenn diese „von weit weniger als 50 Prozent der Wähler unterstützt wird“ (Köppl, S. 167). Zudem ist zu betonen, dass sich auch Koalitionsregierungen nicht zwingend auf mehr als 50 Prozent der Wähler stützen können ${ }^{12}$ und deren akkumulierte Wählerstimmen nur eine (durch Koalitionsvereinbarung) künstlich hergestellte Mehrheit abbilden (Strohmeier, S. 583 f.).

Die „gemäßigte Mehrheitswahl“ ist ein Wahlsystem, mit dem „als primäres Repräsentationsziel eine absolute Parlamentsmehrheit für eine Partei [...] angestrebt wird“ (S. 585).

11 Vgl. auch ausführlich: Gerd Strohmeier, Wahlsysteme erneut betrachtet: Warum die Mehrheitswahl gerechter ist als die Verhältniswahl, in: ZPol, 16. Jg. (2006), H. 2, S. $405-425$.

12 Dies war in Deutschland bei folgenden Koalitionsregierungen der Fall: CDU/CSU-FDP-DP 1949 bis 1953, SPD-FDP 1969 bis 1972, CDU/CSU-FDP 1994 bis 1998, SPD-B’90/Grüne 1998 bis 2002 und SPD-B'90/Grüne 2002 bis 2005. 
Natürlich muss dieses nicht nur angestrebt, sondern in aller Regel auch erreicht werden (Strohmeier, S. 590). Deshalb habe ich ein Wahlsystem vorgeschlagen, bei dem mindestens drei Viertel der Mandate direkt - ohne Verrechnung mit den Listenmandaten - vergeben werden, eine schwächer und eine stärker mehrheitsbildende Variante vorgestellt, und festgehalten, dass für die Festlegung des Verhältnisses zwischen den Direktmandaten und den Listenmandaten das primäre Repräsentationsziel des Wahlsystems maßgebend ist (S. 587). Die Frage, ob das vorgeschlagene Modell tatsächlich in der Lage ist, eine Einparteiregierung hervorzubringen, ist natürlich berechtigt, lässt sich aber nicht (befriedigend) beantworten, indem man - wie Schoen (S. 863 f.) - zunächst (um auf bisherige Wahlergebnisse zurückgreifen zu können) die Gesamtzahl der Parlamentsmandate auf 359 Sitze verringert, dann die Ergebnisse auf der Grundlage des Bundestagswahlergebnisses 2005 durchrechnet und anschließend den Kernbefund dieser Beispielrechnung für ein Parlament mit 598 Mandaten verallgemeinert. Diese Vorgehensweise wirkt elegant, ist aber nicht akzeptabel: Es ist keineswegs von untergeordneter, sondern sogar von entscheidender Bedeutung, dass nicht ein Parlament mit 299 Direktmandaten und insgesamt 359 Sitzen, sondern ein Parlament mit 499 Direktmandaten und insgesamt 598 Sitzen (als mögliche Modellvariante) vorgeschlagen wurde. Schließlich kann nicht davon ausgegangen werden, dass die Ergebnisse beziehungsweise die Mandatsverteilung zwischen CDU/CSU und SPD in 499 völlig neu zugeschnittenen Einerwahlkreisen die gleiche wäre wie in den 299 alten Einerwahlkreisen. Nimmt man auf der Grundlage der bei der Bundestagswahl 2005 erzielten Zweitstimmenergebnisse eine Verteilung der (nach dieser Modellvariante verbleibenden) 99 Listenmandate auf die Parteien vor, erhält die CDU/CSU 35 und die SPD 34 Mandate. Folglich müsste erstere mindestens 265 (53,1 Prozent), letztere mindestens 266 (53,3 Prozent) der insgesamt 499 Direktmandate gewinnen, um die absolute Mehrheit zu erreichen ${ }^{13}$, was sehr gut möglich ist, aber mit einer einfachen Beispielrechnung weder be- noch widerlegt werden kann.

Dies hat auch die in Großbritannien im Dezember 1997 von der Labour-Regierung eingesetzte Jenkins-Kommission beziehungsweise deren Wissenschaftlergruppe erkannt, die ihre Rechnungen auf der Grundlage neu eingeteilter Wahlkreise und aufwändiger Wählerbefragungen durchgeführt hat. Diese haben ergeben, dass das vorgeschlagene „Alternative Vote-Plus System“ in der Regel zu einer Einparteiregierung führen würde. Doch weder dieses noch das von mir vorgeschlagene Wahlsystem können Einparteiregierungen (der stimmenstärksten Partei) garantieren. Schließlich ist kein beziehungsweise kaum ein Wahlsystem in der Lage, das Erreichen des ihm zugrunde liegenden Repräsentationsziels zu garantieren - im Übrigen auch nicht die personalisierte Verhältniswahl mit Sperrklausel ${ }^{14}$. Wahlsysteme wirken - aufgrund der Existenz diverser Kontextfaktoren - nicht immer so, wie sie müssten oder sollten. Aus diesem Grunde ist es nicht beziehungsweise nur begrenzt möglich, die konkrete Wirkungsweise durch einen (kurzen) „Blick über die Grenzen“

13 Dass Köppl (S. 168) „zwischen Wahlkreisen, die für die Entscheidung wichtig, und solchen, die es nicht sind", unterscheidet und den Wählern in ersteren einen ungerechten Einfluss attestiert, ist nicht akzeptabel, da sowohl „marginal seats“ als auch „safe seats“ zur Wahlentscheidung beitragen und letztere erst durch den Wählerwillen zu „Hochburgen“ einer Partei werden.

14 Diese kann - sofern genügend Stimmen aufgrund der Fünfprozentklausel bei der Mandatsverteilung unberücksichtigt bleiben - zu höchst ungerechten beziehungsweise disproportionalen Ergebnissen führen. 
(Köppl, S. 163) zu prüfen. Das politische System Italiens, in dem bei (nur) drei nationalen Parlamentswahlen ein Subtyp der Mehrheitswahl mit proportionaler Zusatzliste Anwendung fand, der dabei sein gewünschtes Ziel zum Teil verfehlte ${ }^{15}$, ist jedenfalls kein (geeigneter) „Testfall“ (Köppl, S. 164) für meinen Vorschlag. Erstens gibt es - wie Köppl (S. 164) am Rande erwähnt - Unterschiede zwischen meinem Vorschlag und dem „italienischen Modell“ (die bei genauerer Betrachtung keineswegs unwesentlich sind). Zweitens gibt es sehr große Unterschiede zwischen Deutschland und Italien (und damit zwischen den Kontextfaktoren). Verwunderlich ist, dass Köppl seine gesamte Replik auf den „Testfall Italien“ stützt, dann aber lapidar anmerkt, dass „eine kurze Auseinandersetzung mit diesem Testfall [...] durchaus sinnvoll gewesen [wäre] “, und zudem einräumt, dass man Italien aufgrund der zu großen parteipolitischen und ideologischen Unterschiede „als Referenzpunkt nicht überstrapazieren" dürfe (S. 165).

Eine bessere „Orientierung“ bietet das bisherige Wahlverhalten in Deutschland - und dies zeigt, dass die Mechanismen der relativen Mehrheitswahl in Einerwahlkreisen hier (wie auch in Großbritannien) durchaus mehrheitsbildend wirken. „Ausufernde“ Versuche, durch „informale Absprachen und / oder Wahlempfehlungen“ (Köppl, S. 166) diesen Effekt „zu unterlaufen", sind in Deutschland erstens nicht zu erwarten und zweitens sehr wohl zu korrigieren, zum Beispiel durch die Einführung der „Alternative Vote“16 (Strohmeier, S. 587). ${ }^{17}$ Das Erreichen des Repräsentationsziels wird natürlich begünstigt, wenn man die mehrheitsbildende Komponente verstärkt, das heißt, wenn man „keine oder sehr wenige Mandate gemäß dem Zweitstimmenanteil verteilte“ (Schoen, S. 864). Verteilt man sehr wenige Mandate nach einem Zweitstimmenanteil, bewegt man sich im Rahmen des vorgeschlagenen Modells. Verteilt man überhaupt keine Mandate nach einem Zweitstimmenanteil, erhält man die relative Mehrheitswahl in Einerwahlkreisen, die ich grundsätzlich nicht - wie Schoen (S. 864) annimmt - verworfen habe. An anderer Stelle habe ich deutlich gemacht, dass diese aufgrund ihrer äußerst starken Konzentrationsfunktion für parlamentarische Regierungssysteme empfehlenswert erscheint, wenn die Mehrheitswahl mit proportionaler Zusatzliste nicht die intendierte Wirkung entfaltet ${ }^{18}$, dass also die relative Mehrheitswahl in Einerwahlkreisen der personalisierten Verhältniswahl mit Sperrklausel grundsätzlich vorzuziehen ist. Ihre Einführung wäre in Deutschland zum gegenwärtigen Zeitpunkt aber aufgrund der erwähnten (S. 585) negativen Effekte für die kleinen Parteien beziehungsweise die Politische Kultur nicht unproblematisch und (daher) auch nur sehr schwer durchsetzbar.

Decker (S. 860) argumentiert, dass die von mir angeblich bemühte Parallele zur Wahlrechtsdiskussion in Großbritannien „völlig an der Sache vorbei geht“. Letztlich tut dies je-

15 Vgl. unter anderem Günther Pallaver, Wahlsystem und Parteiensystem in Italien, in: Österreichische Zeitschrift für Politikwissenschaft, 34. Jg. (2005), H. 1, S. 43 - 60.

16 Die Frage, wie die Mandate in den Einerwahlkreisen vergeben werden (durch die relative Mehrheitswahl oder absolute Mehrheitswahl bzw. „Alternative Vote“), ist aber ähnlich wie die Frage, nach welchem Verfahren die Mandate zugeteilt werden (Hare / Niemeyer oder Sainte-Laguë I Schepers), mit Blick auf den "Grundriss“ des vorgeschlagenen Wahlsystems von nachrangiger Bedeutung (Strohmeier, S. 588).

17 Die von mir vorgeschlagene „gemäßigte Mehrheitswahl“ hätte übrigens selbst nach der Modellrechnung von Schoen mit Ausnahme der Bundestagswahl 2005 bei allen Bundestagswahlen nach der Wiedervereinigung zu einer Einparteiregierung geführt.

18 Gerd Strohmeier, Wahlsysteme erneut betrachtet, a.a.O., S. 423. 
doch Deckers Kritik, da ich keine Parallele zur Wahlrechtsdiskussion in Großbritannien aufzeigen, sondern nur eine weitere Modellvariante der Mehrheitswahl mit proportionaler Zusatzliste vorstellen wollte (das Alternative Vote Plus-System). An der Sache vorbeigehend beziehungsweise sachlich falsch sind auch die weiteren Ausführungen von Decker zur Wahlrechtsdiskussion in Großbritannien beziehungsweise zum Westminster-Modell. ${ }^{19}$ Die Jenkins-Kommission hat nicht versucht, „das Wahlsystem an die veränderten Gegebenheiten anzupassen“ (Decker, S. 860), sondern wollte die beste Alternative zur relativen Mehrheitswahl in Einerwahlkreisen entwickeln. ${ }^{20}$ Entscheidend ist jedoch, dass es nicht gelang, das Mehrheitswahlrecht (geringfügig) zu verändern beziehungsweise (etwas) zu mäßigen. Die Labour-Regierung legte den Vorschlag nicht (wie ursprünglich geplant) dem Volk vor, sondern (ohne großes Aufsehen zu erregen) beiseite - und rührte ihn auch nach der Unterhauswahl 2005 nicht ernsthaft an. Zurzeit ist das Wahlsystem in der britischen Politik kein (ernsthaftes) Thema. Schließlich befürwortet eine große Anzahl britischer Abgeordneter die Mehrheitswahl. ${ }^{21}$ Für viele von ihnen gilt die Verhältniswahl - in den Worten des ehemaligen britischen Außen- und Verteidigungsministers Malcolm Rifkind - als „not democratic" 22 .

Der Versuch, in Deutschland die Mehrheitswahl einzuführen, würde auf Widerstand stoßen. Die vorgeschlagene Wahlrechtsreform könnte letztlich nur gegen den Willen der kleinen Parteien und gegen bestehende Bedenken und (Vor-)Urteile in Teilen der Politik, Gesellschaft sowie Wissenschaft durchgesetzt werden. Sollte die nächste Bundestagswahl zu einer ähnlichen Machtkonstellation wie 2005 führen, wird man aber häufiger, lauter und deutlicher über die Mehrheitswahl diskutieren. Spätestens dann müssen sich die Vertreter der Verhältniswahl intensiver mit den Vorteilen der Mehrheitswahl auseinandersetzen.

19 Seine auf Tony Wright (British Politics. A Very Short Introduction, Oxford 2003) gestützte Behauptung, das Westminster-Modell sei ein „Lehrbuchmodell [...], das auch im Mutterland des mehrheitsdemokratischen Parlamentarismus nicht mehr besteht" (S. 860), ist wohl kaum das Ergebnis einer - von ihm geforderten (S. 857) - seriösen Analyse, sondern vielmehr eine - in seinen Worten - „extrem verkürzte, populistische Sichtweise, der gerade die Politikwissenschaft aufklärerisch entgegenwirken sollte" (S. 861).

20 Dabei sollten verschiedene Ziele berücksichtigt werden, wobei das Ziel einer breite(re)n Stimmen-Mandats-Proportionalität dem Ziel von Einparteiregierungen eindeutig untergeordnet wurde, vgl. The Report of the Independent Commission on the Voting System, London 1998, S. 1 ff.

21 Dies ist auch das Ergebnis von Gesprächen mit Unterhausabgeordneten, die der Verfasser im Rahmen eines von der Robert Bosch-Stiftung ermöglichten Projekts geführt hat.

22 Malcolm Rifkind, Interview, London, 31. Oktober 2007. 